\title{
Review
}

\section{Pulmonary Disease Caused by Non-Tuberculous Mycobacteria}

\author{
Nasstasja Wassilew ${ }^{a, b}$ Harald Hoffmann $^{c, d} \quad$ Claire Andrejak $^{\mathrm{e}}$ \\ Christoph Lange $\mathrm{a}^{\mathrm{a}, \mathrm{b}, \mathrm{f}-\mathrm{h}}$
}

${ }^{a}$ Division of Clinical Infectious Diseases, Research Center Borstel, and ${ }^{b}$ German Center for Infection Research, Clinical Tuberculosis Center, Borstel, ' Institute of Microbiology and Laboratory Medicine, synlab MVZ Gauting,

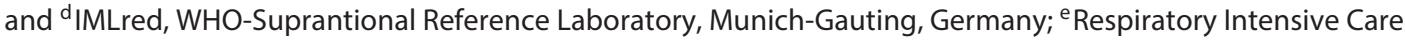
Unit, University Hospital, Amiens, France; ${ }^{\mathrm{f} I n t e r n a t i o n a l ~ H e a l t h / I n f e c t i o u s ~ D i s e a s e s, ~ U n i v e r s i t y ~ o f ~ L u ̈ b e c k, ~ L u ̈ b e c k, ~}$ Germany; ${ }^{9}$ Department of Medicine, Karolinska Institutet, Stockholm, Sweden; h Department of Internal Medicine, University of Namibia School of Medicine, Windhoek, Namibia

\section{Key Words}

Europe $\cdot$ Mycobacterium $\cdot$ Mycobacterium avium/ intracellulare complex . Non-tuberculous mycobacteria . Pulmonary disease

\begin{abstract}
Non-tuberculous mycobacteria (NTM) include more than 160 ubiquitous, environmental, acid-fast-staining bacterial species, some of which may cause disease in humans. Chronic pulmonary infection is the most common clinical manifestation. Although patients suffering from chronic lung diseases are particularly susceptible to NTM pulmonary disease, many affected patients have no apparent risk factors. Host and pathogen factors leading to NTM pulmonary disease are not well understood and preventive therapies are lacking. NTM isolation and pulmonary disease are reported to rise in frequency in Europe as well as in other parts of the world. Differentiation between contamination, infection, and disease remains challenging. Treatment of NTM pulmonary disease is arduous, lengthy, and costly. Correlations between results of in vitro antibiotic susceptibility testing and clinical treatment outcomes are only evident for the Mycobacterium avium complex, M. kansasii, and some rapidly growing mycobacteria. We describe the epidemiology of NTM pulmo-
\end{abstract}

\section{KARGER}

E-Mail karger@karger.com www.karger.com/res nary disease as well as emerging NTM pathogens and their geographical distribution in non-cystic fibrosis patients in Europe. We also review recent innovations for the diagnosis of NTM pulmonary disease, summarize treatment recommendations, and identify future research priorities to improve the management of patients affected by NTM pulmonary disease.

(c) 2016 S. Karger AG, Basel

\section{Introduction}

Non-tuberculous mycobacteria (NTM) are ubiquitous environmental bacteria. Humans are frequently in contact with NTM, as the bacteria live in the soil as well as natural and engineered water systems [1-3]. Most NTM species are non-pathogenic, but some are able to cause human disease [4]. Pulmonary manifestations account for $80-90 \%$ of all NTM-associated diseases [4]. In contrast to pulmonary tuberculosis (TB), direct human-tohuman transmission of NTM has infrequently been reported [5-7]. Mycobacterium abscessus, M. avium complex, $M$. kansasii, $M$. malmoense, and $M$. xenopi are the clinically most important species. Depending on the causative NTM species, the clinical course and treatment
C 2016 S. Karger AG, Basel

0025-7931/16/0915-0386\$39.50/0
Christoph Lange, MD

Division of Clinical Infectious Diseases Research Center Borstel, Parkallee 35 DE-23845 Borstel (Germany)

E-Mail clange@fz-borstel.de 
response of NTM pulmonary disease (NTM-PD) can be very variable [8]. During the last three decades, an increasing incidence of pulmonary NTM isolation has been observed in Europe and several other regions worldwide [8-13]. The increase seems to be associated with the declining incidence of TB in countries with a higher socioeconomic standard [14]. Several factors may contribute to the emergence of NTM-PD, including an aging population with chronic lung diseases and advances in radiological diagnostics that have improved the identification of pulmonary abnormalities [15]. Population-based studies focusing on the demographic change in NTM-PD remain scarce.

In this review we portray NTM-PD from a European perspective, concentrating on the epidemiology and description of NTM species as emerging pulmonary pathogens in non-cystic fibrosis patients. Consensus recommendations for the management of NTM in individuals with cystic fibrosis have recently been published $[16,17]$. We review recent diagnostic innovations and advances in NTM-PD case management which are not specific to Europe alone. Disseminated NTM diseases [as e.g. in people living with human immunodeficiency virus (HIV)], NTM-associated lymphadenitis in children, or other extrapulmonary manifestations of NTM infections are not the focus of this review.

\section{Methods}

A review of the available literature was accomplished by searching electronic databases including MEDLINE and PubMed, using the following key words: 'non tuberculous mycobacteria', 'nontuberculous mycobacteria', 'Mycobacterium avium complex', 'M. abscessus', 'M. kansasii', 'M. xenopi', and 'M. malmoense'. In addition, reference lists from review articles and guidelines were handsearched for relevant articles.

\section{Epidemiology and Emerging Pathogens}

The genus Mycobacterium was introduced in 1896, a decade after the discovery of $M$. tuberculosis as the causative pathogen of TB [18]. A total of 182 taxa (169 species and 13 subspecies) have been assigned to the genus so far [2], including several heterotypic synonyms. Excluding synonymic taxa, 165 species and 8 subspecies can be distinguished. Some taxa are phylogenetically closely related and are referred to as 'complexes'. It is important to note that those complexes are taxonomically poorly defined, and different authors might subsume different taxa under the respective complex denominations. However, with a certain degree of consensus, most microbiologists consider the M. avium/intracellulare complex (MAC) to consist of at least 10 species, i.e. M. avium, M. intracellulare, M. arosiense, $M$. bouchedurhonense, M. chimaera, $M$. colombiense, M. marseillense, M. timonense, M. vulneris, and $M$. yongonense, as well as 4 subspecies, i.e. M. avium subsp. avium, $M$. avium subsp. silvaticum, $M$. avium subsp. hominissuis, and M. avium subsp. paratuberculosis, which have high genetic similarity [19-22]. Thirty to 60 NTM species are repeatedly recovered from clinical specimens, while the majority of NTM have only rarely if ever been observed in clinical settings [23]. As NTM isolation or disease is not notifiable to public health authorities in Europe, epidemiological surveys are not routinely performed for NTM-PD.

In some cases, differentiation of species and subspecies may have a clinical impact. For example, it has been demonstrated that specific MAC species may have varying degrees of virulence leading to different clinical courses [24]. M. bolletii and M. massiliense were proposed as 2 new species of the M. abscessus complex in 2006 [25], but in 2011 it was suggested that both species be reunified under the single subspecies M. abscessus bolletii [26]. Both species and subspecies denominations are valid today. For clinicians, however, the identification of $M$. $a b$ scessus subsp. massiliense (former M. massiliense) is helpful. While both of the other M. abscessus subspecies harbour the inducible erythromycin ribosome methyltransferase 41 (erm41) gene, which enables them to intracellularly inactivate macrolides leading to various degrees of resistance after exposure to these antibiotics, the gene is not functional in M. abscessus subsp. massiliense. Consequently, this species is generally susceptible to macrolides [27, 28].

M. gordonae is among the Mycobacterium species most frequently recovered from environmental samples worldwide. Recovered from a clinical sample, it is mostly considered not to be clinically relevant, even though disease may occur. Online supplementary table S1 (see www. karger.com/doi/10.1159/000445906 for all online supplementary material) gives examples of the differential relevance of some Mycobacterium species if isolated from clinical specimens [29].

MAC is by far the most frequently encountered group of pathogens of NTM-PD in European countries [30-32], with $M$. avium subsp. hominissuis being the predominant subspecies recovered from human biospecimens $[33,34]$. A recent international prevalence survey showed marked regional differences in the isolation of mycobacterial spe- 
cies [30]. MAC was the species most frequently recovered in the majority of European countries, though M. xenopi was more commonly isolated in Hungary (49 vs. 16\%) and M. kansasii in Poland and Slovakia (35 and 36\% vs. 23 and 28\%, respectively). Rapidly growing Mycobacterium species were more common in the UK and in Greece as compared to MAC ( 44 and $46 \%$ vs. 22 and 36\%, respectively). In London, UK, the predominant NTM species related to disease in HIV-seronegative individuals was $M$. kansasii [35]. This is in contrast to the high prevalence of M. abscessus complex on the North American, Asian, and Australian continents, where it is the second most frequent NTM after MAC causing NTM-PD [36-38]. In the majority of European countries, $M$. xenopi (which represents $<0.01 \%$ of isolated NTM), followed by $M$. kansasii and $M$. malmoense, is more frequently encountered in NTM-PD than is M. abscessus in non-cystic fibrosis patients $[8,9,39-42]$.

The prevalence of NTM species has changed over time in Europe [34]. For example, during the past decades, infections with $M$. xenopi have increasingly been observed in Central, Southern, and Western Europe, while they appear to be almost absent in Northern Europe [34]. In contrast, in Northern Europe M. malmoense infections are emerging [30, 34, 39, 43]. It appears that specific environmental factors related to soil and water increase the risk of pulmonary NTM disease $[32,44]$, but they do not comprehensively explain the geographic variation of different species [45].

Incidence rates of NTM-PD in Europe range from 0.2 to $2.9 / 100,000$ population. In Croatia, the overall estimated age-standardized annual incidence of probable NTMPD was $0.2 / 100,000$ population (2006-2010), with a difference between the coastal $(0.4 / 100,000)$ and the continental region $(0.2 / 100,000)$ [40]. In Scotland, the mean incidence rate of NTM episodes was reported to be $2.4 / 100,000$ population, with no clear trend over the years 2000-2010 [41], while increasing NTM isolation incidence rates were observed in another study from the UK, rising from 0.9 to $2.9 / 100,000$ population (1995-2006), and the incidence of NTM-PD rose in the English region of Leeds from 0.8 to 2.0/100,000 population (1995-1999) $[9,46]$. In Denmark, the NTM-PD incidence increased from 0.6 to $1.5 / 100,000$ population-years from 2003 to 2008 [39]. In Germany, the overall age-adjusted rates of pulmonary NTM infection-associated hospitalizations increased from 0.7 to $1.1 / 100,000$ population [11]. Increases in the incidence of NTM-PD in different parts of Europe are likely related to demographic changes with accompanying risk factors for lung health [8].

\section{Population Structure}

Pre-existing chronic lung diseases, especially COPD, asthma, and bronchiectasis, are the main risk factors for NTM-PD in Europe [39, 47-50]. In contrast to the USA, fibrocavitary disease or consolidating infiltrates are more common than nodular bronchiectatic NTM-PD in Europe $[47,51]$. A considerable proportion of patients with NTM-PD in Europe have no detected underlying lung disease or immunodeficiency $[9,39,52]$.

\section{Clinical Presentation}

Clinical symptoms in patients with NTM-PD may be indistinguishable from TB or other respiratory diseases, including lung cancer [4]. Patients typically present with fatigue, fever, weight loss, asthenia, and/or anorexia [4, 47]. Respiratory symptoms may consist of cough, sputum production, haemoptysis, or dyspnoea and can be secondary to various respiratory tract or parenchymal diseases $[4,47]$. A plain chest radiograph may be inadequate for evaluating radiological features. High-resolution computed tomography scans more precisely demonstrate the extent of parenchymal lung damage, particularly by visualization of nodular bronchiectatic or small cavitary lesions $[4,53]$.

Recent studies have suggested that the nodular bronchiectatic pattern is the predominant form of MAC-induced disease, accounting for approximately $50 \%$ of cases and ranging from 43 to $79 \%$ in Canada, the USA, Korea, and Japan. In contrast, the rates of fibrocavitary and unclassified forms in the same studies ranged from 17 to $40 \%$ and from 6 to $39 \%$, respectively [54-57]. Few studies from Europe used the same radiopathological classifications, and comparative studies of the clinical spectrum of NTM-PD manifestations between Europe and other areas of the world are missing.

It appears that the majority of cases with MAC-PD in Europe fulfil neither the criteria for fibrocavitary nor for nodular bronchiectatic disease, and that the manifestations of the disease vary. Van Ingen et al. [47] reported that patients with MAC-PD more often had cavitary than nodular bronchiectatic disease in the Netherlands. Among patients with MAC-PD in France $[47,52]$ there were lower rates of fibrocavitary than of nodular bronchiectatic disease, which is similar to the results of recent surveys in the USA and South East Asia [53-58]. Coinfection with MAC and M. abscessus subsp. abscessus occurs and may be associated with new or enlarged cavities in 

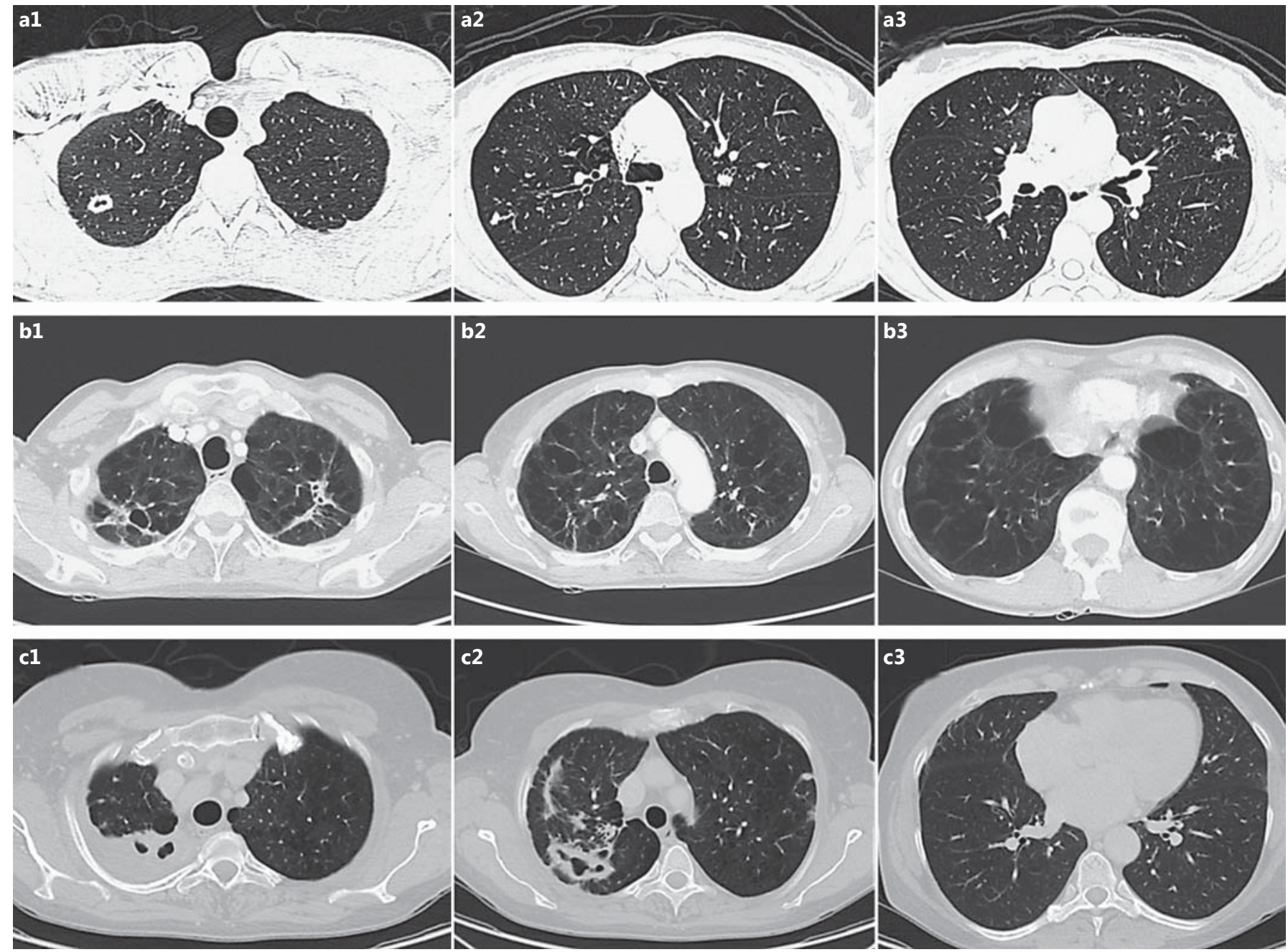

Fig. 1. Thoracic CT images from patients with NTM-PD. a A 48-year-old non-smoking woman with pectus excavatum and a 4-month history of fever and night sweats, a small cavitation in the right upper lobe (a1) and nodular bronchiectatic infiltrates in the right (a2) and left (a3) upper lobes. Acid-fast bacilli were detectable on sputum smears. Sputum culture repeatedly grew $M$. intracellulare. b A 63-year-old woman with a smoking history of $>50$ pack-years of cigarettes, COPD GOLD stage 3 lung disease, chronic productive cough, night sweats, and weight loss. Fibrous scaring and traction bronchiectasis can be seen in both upper lobes (b1, b2), as can centrilobular emphysema (b2, b3). Acid-fast bacilli were detectable on sputum smears, and sputum cultures grew $M$. abscessus on 2 different occasions. c A 57-year-old woman with a smoking history of 45 pack-years of cigarettes and COPD GOLD stage 3 lung disease. She presented with productive cough, night sweats, and weight loss. Acid-fast bacilli were detectable on sputum smears. Sputum and bronchoalveolar lavage cultures grew $M$. malmoense. Thoracic imaging revealed pleural thickening (c1), dense infiltrates (c1), and a cavity (c2) in the upper right lobe, as well as peripleural nodular infiltrations in the left upper lobe. No further abnormalities were seen on thoracic CT (representative image: c3). Although all 3 images series are typical for NTM-PD, species identification is not possible by radiological imaging. Clinical and microbiological information is required together with imaging results to arrive at a decision for treatment. case of additional M. abscessus-PD [59]. This underlines the necessity of regular microbiological and clinical follow-ups to determine which patients truly have $M$. $a b$ scessus-associated lung disease during the course of MAC-PD.
In several studies from different regions, infections with rapidly growing mycobacteria were less frequently and infections with $M$. kansasii, M. malmoense, or $M$. xenopi more frequently associated with fibrocavitary disease $[43,44,54,55,58,60-65]$ (fig. 1), demonstrating a 
Table 1. Wolinsky's clues: support for the diagnosis of NTM-PD [adapted from 75]

\begin{tabular}{llll}
\hline Clue & Supporting a diagnosis of NTM-PD & Commentary \\
\hline 1 Quantity of growth & $\begin{array}{l}\text { Moderate or heavy bacillary growth from } \\
\text { sputum or BAL specimen }\end{array}$ & $\begin{array}{l}\text { Relevant to NTM-PD, since moderate or heavy growth } \\
\text { of mycobacteria from bronchopulmonary specimens is } \\
\text { usually associated with disease }\end{array}$ \\
\hline $\begin{array}{l}\text { Repeated isolation } \\
\text { of the same species }\end{array}$ & Repeated isolation of the same organisms & $\begin{array}{l}\text { Single, random isolations often connote contamination; } \\
\text { the indication for treatment of NTM-PD should not be } \\
\text { based on one unconfirmed isolation of an NTM species }\end{array}$ \\
\hline $\begin{array}{l}\text { Site of positive } \\
\text { specimen }\end{array}$ & $\begin{array}{l}\text { Isolation of NTM from a sterile body site, } \\
\text { e.g. lung tissue }\end{array}$ & $\begin{array}{l}\text { Less relevant to NTM-PD, since the diagnosis is mostly } \\
\text { based on BAL or sputum examinations }\end{array}$ \\
\hline $\begin{array}{l}\text { Identified NTM } \\
\text { species }\end{array}$ & $\begin{array}{l}\text { Isolation of } M \text {. abscessus, } M . \text { kansasii, and } \\
\text { M. malmoense from a bronchopulmonary } \\
\text { specimen is usually related to PD }\end{array}$ & $\begin{array}{l}\text { M. gordonae is among the NTM species most frequently } \\
\text { isolated from bronchopulmonary specimens but is } \\
\text { almost always not related to NTM-PD; treatment of } M . \\
\text { gordonae infection should only be initiated if other clues } \\
\text { are present }\end{array}$ \\
\hline $\begin{array}{l}\text { Host risk factors } \\
\text { BAL = Bronchoalveolar lavage. }\end{array}$ & $\begin{array}{l}\text { Risk factors for NTM-PD include } \\
\text { bronchiectasis, COPD, and other chronic } \\
\text { lung diseases }\end{array}$ & $\begin{array}{l}\text { Cave: chronic lung disease also predisposes to } \\
\text { colonization with NTM }\end{array}$ \\
\hline
\end{tabular}

species dependency of the different forms of NTM disease.

Hypersensitivity pneumonitis due to mycobacteria (also called 'hot tub lung') is a hypersensitivity reaction occurring in patients who are exposed to MAC antigens, e.g. from sources such as indoor hot tubs [66] or colonized showers $[67,68]$. Occupational exposure to $M$. immunogenum has also been described $[69,70]$. The diagnosis is established based upon clinical, radiological, and immunological criteria plus the optional presence of corresponding mycobacteria in the respiratory system. There is a continuing debate on optimal management of these patients [71]. The fact that a high rate of patients have been reported to be cured by avoidance of the antigen and/or corticosteroid therapy without antimycobacterial therapy suggests that hot tub lung is more likely a form of hypersensitivity pneumonitis than an infectious disease [72-74].

\section{Diagnosis}

In the early 1980s, Emanuel Wolinsky, a pioneer in the field of NTM diseases, proposed 5 criteria to distinguish clinically relevant NTM disease from presence of NTM without relevant clinical correlates; those were (1) medi- um-to-heavy growth of NTM in culture, (2) repeated isolation, (3) an origin of a positive specimen from sites with little or no contact with the environment, (4) a mediumto-high probability that the isolated NTM species causes disease, and (5) the presence of risk factors predisposing to NTM disease (table 1) [75]. The American and British Thoracic Societies proposed 3 similar though simplified criteria for the establishment of the diagnosis of NTM disease: (1) compatible correlates in a radiograph or CT scan of the thorax, including bronchiectasis, infiltrates, multiple nodules, multifocal bronchial disease, and cavities, plus (2) compatible clinical symptoms and exclusion of other diseases with similar symptoms and radiological signs, including TB, plus (3) at least 2 sputum samples which are positive on culture from 2 separate expectorated samplings or 1 positive culture from at least 1 bronchial wash or lavage (both of which are only relevant for patients with nodular bronchiectatic disease, who do not expectorate sputum) or isolation of mycobacteria from a sterile site, including lung tissue obtained by transbronchial or open lung biopsy (online suppl. table S2) $[4,76]$. It should be noted that these diagnostic criteria were developed with respect to disease caused by MAC, M. kansasii, or M. abscessus and may have to be further adapted to nodular bronchiectatic as compared to fibrocavitary disease. 
In addition to the current diagnostic guidelines $[4,76]$, molecular tests for species identification and antibiotic susceptibility testing (AST) should be part of any diagnosis of NTM-PD where available [24, 77]. Smear microscopy of respiratory secretions yields positive results in approximately half of patients with probable or ascertained disease [38, 47, 78]. Acid-fast bacilli are more likely to be visible in patients fulfilling the ATS criteria; they can be regularly detected in patients presenting with cavities and are only rarely seen in patients with nodular bronchiectatic NTM-PD [58]. Smear microscopy is particularly valuable in combination with nucleic acid amplification tests (NAAT) for M. tuberculosis. All commercial, CEmarked (a mandatory conformity marking for certain products sold within the European Economic Area) NAAT for TB share negative predictive values higher than $99.5 \%$ in smear-positive samples under low-TB prevalence conditions $[79,80]$. Consequently, NTM are identified with very high probability in cases with acidfast bacilli observable on sputum smear microscopy and negative $M$. tuberculosis NAAT results from the same sample (online suppl. table S3).

Few CE-marked commercial NAAT allow for the direct detection of NTM DNA in respiratory secretions, including $\operatorname{artus}^{\circledR}$ Mycobac. diff. LC PCR (Qiagen, Hilden, Germany), Speed-oligo ${ }^{\circledR}$ Direct Mycobacterium tuberculosis (Vircell, Granada, Spain), Geno-Sen's MTC/ MOTT Real Time PCR (Corbett Research, Mortlake, N.S.W., Australia), and GenoType ${ }^{\circledR}$ Mycobacteria Direct (Hain Lifescience, Nehren, Germany). While the artus ${ }^{\circledR}$ assay differentially detects DNA of only the M. tuberculosis and M. avium complexes on a real-time PCR platform, Speed-oligo ${ }^{\circledR}$ and Geno-Sen's detect $M$. tuberculosis complex and the genus Mycobacterium by reverse hybridization of PCR products in a dipstick format and a real-time PCR assay, respectively, and GenoType ${ }^{\circledR} \mathrm{My}$ cobacteria Direct detects the genus Mycobacterium plus 4 NTM species [81]. By the end of 2016, the last assay will most likely be replaced by a new generation of GenoType ${ }^{\circledR} \mathrm{CM}$, a line probe assay which will be adjusted for direct detection of up to 13 NTM species in clinical specimens (Hain Lifescience, pers. commun.). Some other assays like the CapitalBio Mycobacteria Real-Time PCR Detection Kit (CapitalBio Corporation, Beijing, China) or the REBA Myco ID (YD Diagnostics, Yongin, South Korea) are offered by Eastern Asian companies and are not yet CE marked. The REBA Myco ID assay was developed for the differentiation of 20 species in culture, but it was demonstrated also to be a useful tool for the identification of NTM in smear-positive specimens with $98 \%$ overall concordance with culture [82]. Due to the ubiquity of NTM, all these assays suffer from the same shortcoming, i.e. that the qualitative detection of NTM DNA in the respiratory tract does not prove any causal association of NTM with disease. That is most likely why none of these assays has ever reached widespread acceptance. But following Wolinsky's postulates [75], the rapid identification of those species which are most likely associated with clinical correlates should be considered as evidence of NTM-PD. Therefore, molecular assays directly applied to clinical specimens should be able to detect the most relevant Mycobacterium species.

Most NTM grow under the same culture conditions as M. tuberculosis. While British guidelines demand the combination of a liquid medium [e.g. Middlebrook 7H9 in MGIT ${ }^{\circledR}$ (Becton, Dickinson and Company, Franklin Lakes, N.J., USA), BactAlert (bioMérieux, Marcy l'Etoile, France), or VersaTrek (Trek Diagnostic Systems, Independence, Ohio, USA)] with either Löwenstein-Jensen or a Middlebrook agar (7H10 or 7H11), German guidelines demand 2 solid media (Löwenstein-Jensen or Ogawa plus Middlebrook agar or Stonebrink or Gottsacker or Coletsos medium) [76, 83, 84]. Whereas a benefit from the combination of a solid and a liquid medium for the sensitivity ( $>10 \%$ increase) of culture has been proven in previous studies, the combination of 2 solid media might be more useful for growth of M. bovis than of NTM [85]. The automated reader of the Bactec MGIT 960 machine might fail to detect some fastidious NTM species [86]. German guidelines claim to address this weakness by (1) visual control of every negative MGIT tube for faint growth, (2) prolonged incubation of negative cultures if smear microscopy has been positive, and (3) molecular tests for the presence of NTM in culture if the clinical signs are typical of mycobacteriosis but culture remains negative [84]. Some NTM species such as M. genavense or M. haemophilum, though practically always pathogenic when isolated from clinical specimens, only grow in culture after a prolonged incubation time or after the addition of special growth factors $[87,88]$. Once growth is detected, $M$. tuberculosis should immediately be excluded by use of antigen-based rapid tests [e.g. the Capilia TB assay (TAUNS, Izunokuni, Japan) or the MGIT TBc ID Test (Becton, Dickinson and Company], PCR, or line probe assays [89]. If NTM are identified, they should be taxonomically differentiated to the level of complex, species, or subspecies depending on their clinical relevance. Most European laboratories initially use reverse hybridization line probe assays such as Genotype CM/AS (Hain Lifescience) or InnoLiPA Mycobacteria (Innogenetics, 
Ghent, Belgium), which allow for the differentiation of up to 13 species in one assay. Very recently, new commercially available spectral reference libraries have been issued for the differentiation of NTM using matrix-assisted laser desorption/ionization time-of-flight (MALDI-TOF) mass spectrometry [90]. In this recent evaluation, the results correlated in $97.4 \%$ of the cases with the genetic taxon identification based on $16 \mathrm{~S}$ rRNA gene and subsequent $r p o B$ or $h s p 65$ sequencing. The preparation protocol of the test matrix as requested by the manufacturer is quite labour intensive. A new, much easier protocol has been validated rendering this method more feasible for routine practice [91]. While molecular tests, i.e. line probe assays, gene sequencing, and PCR assays, are all in the same range of costs depending on the on-site staff and equipment available, cost-efficiency studies of MALDITOF for the differentiation of mycobacteria are still pending. In virtually all European laboratories, molecular assays have displaced biochemical tests or mycolic acid chromatography for the differentiation of mycobacteria [92]. Consequently, the degree of taxonomical diversification is much higher in contemporary studies, rendering comparative observations with regard to the emergence or disappearance of certain species unreliable.

A variety of methods have been proposed for AST of NTM, including micro- and macrodilution tests, the E test, and proportional- and absolute-concentration methods $[93,94]$. While the American Clinical and Laboratory Standards Institute (CLSI) provides clear guidelines as to when and how to use AST of NTM and how to interpret minimal inhibitory concentrations, British guidelines do not mention AST, the European Committee on Antimicrobial Susceptibility Testing (EUCAST) provides breakpoints only for 2 antimycobacterial drugs (delamanid and bedaquiline) which are not recommended for the treatment of NTM-PD at present, and German guidelines advise caution with reference to missing evidence for the clinical relevance of AST results from large cohorts [76, 84, 95-97]. Following CLSI standards, evidence of in vitro susceptibility and clinical outcome is satisfactory for MAC, M. kansasii, M. marinum, and M. abscessus subsp. massiliense as long as broth microdilution AST are used. E tests suffer from high intra- and inter-laboratory variability, and the proportional method on solid media or in MGIT has its breakpoints not yet defined for NTM. In view of the emergence of NTM-PD with $M$. abscessus complex, European authors recommend determination of the inducibility of the erm 41 gene by exposing $M$. $a b$ scessus isolates to clarithromycin for 14 days [93]. Yet, with the increasing clinical importance of NTM disease, evaluation of the clinical relevance of AST results from NTM should receive greater attention by both mycobacteriology research centres and grant donors of respective clinical trials.

\section{Management}

In clinical practice, patients with severe and/or progressive NTM-PD are considered candidates for antibiotic multidrug therapy $[4,76]$. The ATS/IDSA diagnostic criteria help to differentiate between patients requiring and patients not requiring treatment, but they are probably better applicable to patients with nodular bronchiectatic disease than to those with fibrocavitary disease. Particularly in the USA, where the nodular bronchiectatic form of NTM disease predominates, a substantial proportion of more than $40 \%$ of patients with NTM might require antimycobacterial treatment based on the individual judgement of the physician in charge [98]. When patchy consolidation is the only presentation on thoracic imaging, the decision to initiate treatment is less stringent.

Treatment recommendations are mostly based on expert opinions and traditions [99]. The scientific evidence base for most recommendations is narrow and is largely derived from retrospective cohort studies, drug susceptibility surveys, or animal experiments [100-102]. Only one prospective, placebo-controlled clinical trial on pulmonary infections caused by MAC is available, which compares the treatment outcome of pulmonary MAC infection with and without additional aminoglycoside in addition to the standard treatment regimen [103]. This trial is particularly interesting for European countries, since half of these patients presented with fibrocavitary disease. Clinical improvement was better in the streptomycin group with regard to microbiological findings, underlying respiratory diseases, and radiological findings, but the difference was not significant [103].

Surgery is an important option for patients with severe NTM-PD who have a poor response to medical therapy, localized cavitation, or severe nodular bronchiectatic disease; however, the complication rate can be high [54]. Two different retrospective analyses showed a persistent sputum culture conversion rate after surgery and postsurgical medical treatment in 81 and $88 \%$ of patients, respectively $[54,104]$. If surgery is feasible, it should be considered for patients with persistent culture positivity after 6 months of medical treatment [104]. 
An important shortcoming of management of patients with NTM-PD is that standardized endpoints for treatment outcomes are lacking [105]. Outcome objectives may vary from sputum culture conversion to simple improvement of clinical and radiological signs.

The cornerstones of most anti-NTM drug regimens are macrolides, but there are exceptions such as M. kansasii-PD. Table 2 lists the drugs used in treatment of NTM-PD. Suggested treatment regimens are shown in table 3. Clarithromycin and amikacin are the only drugs with a shown correlation between in vitro drug susceptibility and in vivo efficacy in MAC-PD [106-109]. Clarithromycin is usually combined with some rifamycin and with ethambutol [4]. No data are available that suggest any superiority of either clarithromycin or azithromycin in the treatment of pulmonary MAC disease [110]. For MAC-PD, far more data are available for clarithromycin than for azithromycin, but in clinical practice azithromycin is frequently used due to its preferable dosing schedules and better gastrointestinal tolerance. Azithromycin also is considered less prone to interaction with rifamycins than is clarithromycin [111].

Induction of cytochrome $\mathrm{P} 450$ by rifampicin may lower macrolide blood levels below inhibitory concentrations [111]. It is not well understood, however, why patients with NTM-PD might still respond clinically [112, 113]. Whether therapeutic drug monitoring improves treatment outcomes in MAC-PD is currently under investigation $[113,114]$.

In HIV-seropositive individuals, rifabutin is often preferred over rifampicin because of less frequent drug-drug interactions with antiretroviral therapies. In case of treatment with rifabutin, azithromycin should be the macrolide of choice $[115,116]$.

In nodular bronchiectatic MAC-PD, intermittent (3-times-weekly) therapy with a macrolide, a rifamycin, and ethambutol has been suggested as a reasonable initial treatment regimen $[117,118]$. This treatment is common in the USA but less popular in Europe. In case of MAC in vitro drug resistance or adverse effects, the alternative drugs are amikacin, streptomycin [119], or, with less clinical evidence, clofazimine $[120,121]$. Patients with severe MAC-PD or fibrocavitary disease in particular might profit from aminoglycoside treatment in addition to the standard regimen in the intensive treatment phase [103, 122].

Results from recent trials support inhalation of aerosolized amikacin [123], especially liposomal amikacin [124]. In vitro synergistic activity against NTM was reported for clofazimine and amikacin [125]. The impact of linezolid on treatment outcome in NTM-PD remains unclear $[126,127]$. A high frequency of adverse drug events in long-term therapy restricts the use of linezolid as part of a treatment regimen for patients with severe disease and distinct patterns of antibiotic resistance or intolerance to other drugs [128].

Rifampicin is the backbone of treatment for M. kansasii-PD (table 3) [129]. The preferred combination partners for multidrug regimens are ethambutol plus either isoniazid or a macrolide [130]. Some authors have proposed to shorten the treatment period for M. kansasii-PD to 12 [130] or even 9 months $[76,131]$ compared to the 12 months after sputum culture conversion recommended by the ATS/IDSA [4]. Patients with rifampicin-resistant $M$. kansasii-PD might require a 3- to 4-drug regimen based on in vitro susceptibilities, including isoniazid, moxifloxacin, ethambutol plus a macrolide or aminoglycoside. Some experts suggest extending the duration of treatment to 18 months in this situation.

The results of various treatment regimen evaluations for M. malmoense-PD were inconclusive in 2 trials conducted by the British Thoracic Society $[132,133]$. The current recommendation for the treatment of M. malmoense-PD is a combination therapy including a rifamycin, ethambutol, and a macrolide, with moxifloxacin being an alternative to the macrolide in case of adverse events or failing therapeutic response (table 3) [119].

In the absence of sufficient data on clinical outcomes of $M$. xenopi infections upon antimycobacterial therapy, recommendations must be formulated with caution (table 3). A combination therapy with a rifamycin and ethambutol together with a macrolide (possibly also adding moxifloxacin) for 12 months beyond the date of culture conversion has been proposed [119]. An ongoing randomized clinical trial in France compares the efficacy and tolerance of moxifloxacin- and clarithromycin-containing regimens.

While M. simiae infection relatively often leads to PD in some parts of the USA, this is much less commonly the case in Europe. As with many other mycobacteria causing NTM-PD, the optimal treatment regimen for M. simiae$\mathrm{PD}$ is not known. In vitro DST reveals an antibiotic drug resistance against many different compounds. The choice of drugs for treatment should be based on DST results. Combination drug regimens often include amikacin in association with 2-3 other drugs [134]. A combination of amikacin and clofazimine has synergy in vitro against $M$. simiae isolates, suggesting the use of these 2 drugs as part of a regimen [135]. Trimethoprim/sulfamethoxazole is sometimes part of a treatment regimen in M. simiae-PD. 


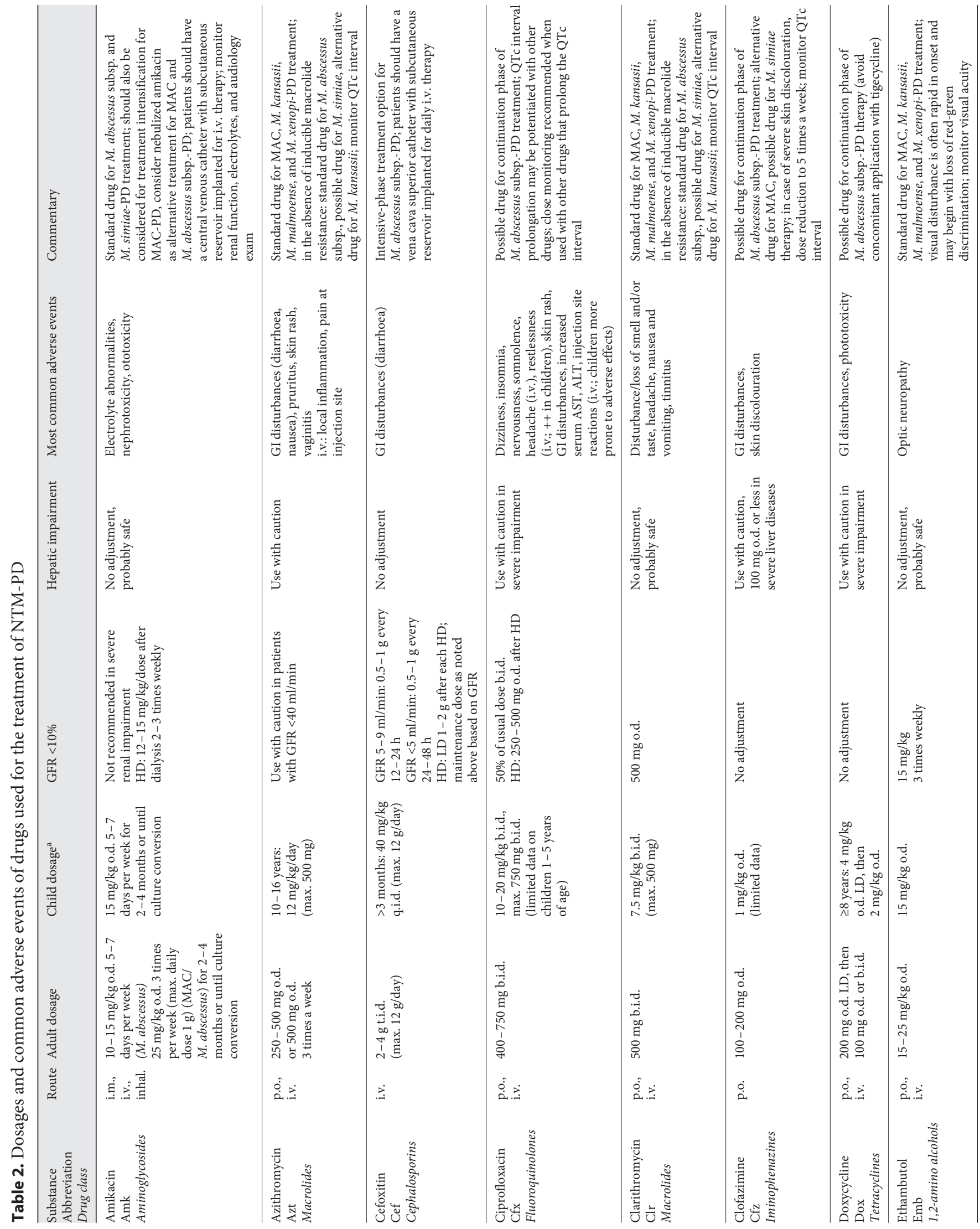




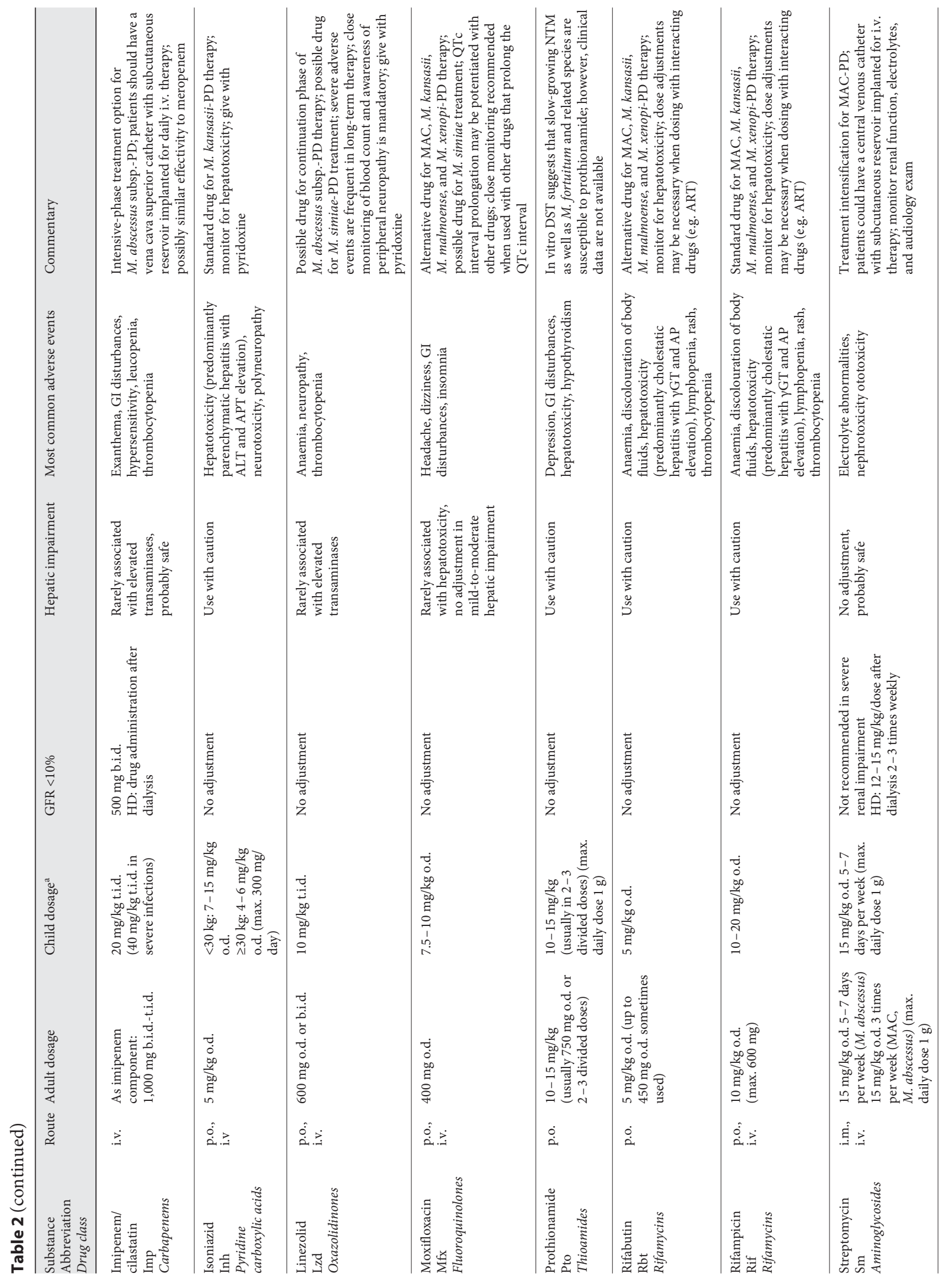




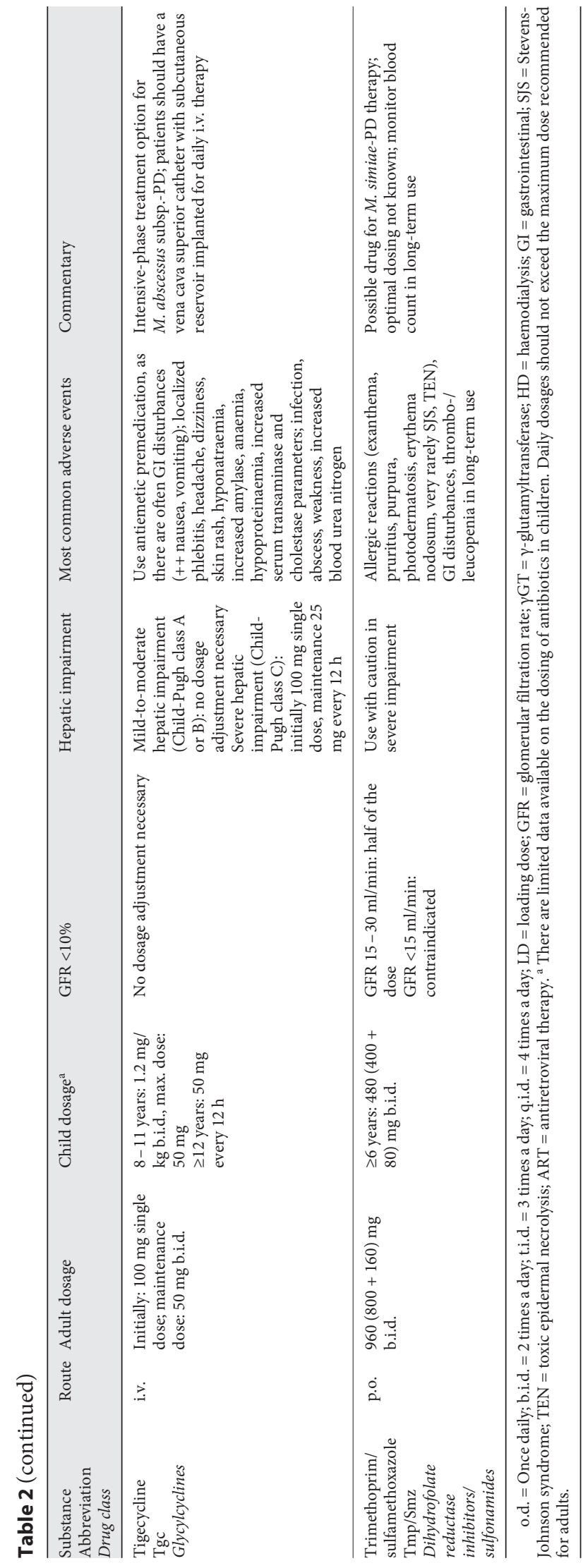

In M. abscessus-PD, sustained culture conversion and clinical improvement can often not be achieved with any presently available drugs independently of the chosen regimen [136]. Symptom control and microbial suppression may be the best achievable goal of therapy.

In vitro drug resistance to multiple antimycobacterial antibiotics is common in M. abscessus [136]. The mechanisms leading to intrinsic or acquired resistance are particularly complex and not necessarily induced by previous exposure to antibiotics $[137,138]$. Because of its variability in susceptibility and its potential to induce macrolide resistance [27], AST is generally recommended. Still, the in vitro-in vivo correlation between drug resistance testing and clinical outcome in M. abscessus-PD is poor [139], and the role of AST in achieving successful treatment remains uncertain $[119,140,141]$. Several studies applying molecular tools to distinguish $M$. abscessus subsp. abscessus from $M$. abscessus subsp. massiliense have shown a better outcome for patients infected with M. abscessus subsp. massiliense treated with a macrolide [44, 141-143]. Measuring inducible macrolide resistance has become a standard laboratory test, based on either erm41 gene sequence analysis or phenotypic testing with prolonged incubation. Currently, there is no consensus on $M$. abscessus complex treatment, except for the use of a macrolide in the absence of resistance, e.g. due to the erm41 gene. A possible recommendation for the treatment of $M$. abscessus-PD consists of two phases [4, 119, 144]. The first one is the intensive phase, with at least two injectable agents (amikacin plus cefoxitin, imipenem, or tigecycline) plus three orally administered drugs (e.g. ciprofloxacin, doxycycline, and linezolid). Following culture conversion, treatment is usually continued with the orally available drugs only. Clofazimine should be considered as an alternative drug in the treatment of $M$. abscessus complex disease [144]. In vitro studies have shown a promising synergistic effect when used concomitantly with amikacin $[125,145]$. In a small case series, bedaquiline had a non-sustained treatment effect against $M$. $a b$ scessus [146]. If the results of prolonged DST suggest a susceptibility of $M$. abscessus species to macrolides, one of the macrolides, preferably azithromycin, should always be part of the drug regimen. Clarithromycin might induce macrolide resistance in the M. abscessus complex more frequently than azithromycin [147], but this finding is not consistent [148]. The choice of companion drugs that will protect the macrolide from resistance is controversial. If feasible, surgery should always be considered for M. abscessus-PD [140]. 
Table 3. Drug treatment of NTM-PD [adapted from 119, 144]

\begin{tabular}{|c|c|c|}
\hline & Recommended regimen & Alternative drugs \\
\hline $\begin{array}{l}\text { M. abscessus subsp. } \\
\text { abscessus and subsp. } \\
\text { bolletii (consider } \\
\text { inducible macrolide } \\
\text { resistance) }\end{array}$ & $\begin{array}{l}2 \text { drugs i.v. (IP only): amikacin plus either cefoxitin or imipenem } \\
\text { or tigecycline } \\
\text { plus } \\
\mathbf{3} \text { drugs p.o. (IP and CP) of the following: macrolide }{ }^{1} \text { (in case of } \\
\text { absence of inducible macrolide resistance), clofazimine, linezolid, } \\
\text { ciprofloxacin, doxycycline (not together with tigecycline) }\end{array}$ & $\begin{array}{l}\text { Consider nebulized (liposomal) } \\
\text { amikacin (IP and CP) }\end{array}$ \\
\hline $\begin{array}{l}\text { M. abscessus subsp. } \\
\text { bolletii, former subsp. } \\
\text { massiliense (usually no } \\
\text { inducible macrolide } \\
\text { susceptibility) }\end{array}$ & $\begin{array}{l}2 \text { drugs i.v. (IP only): amikacin plus either cefoxitin or imipenem } \\
\text { or tigecycline } \\
\text { plus } \\
3 \text { drugs p.o. (IP and CP): macrolide }{ }^{1} \text { (in case of absence of inducible } \\
\text { macrolide resistance) plus } 2 \text { of the following: clofazimine, linezolid, } \\
\text { ciprofloxacin, doxycycline (not together with tigecycline) }\end{array}$ & $\begin{array}{l}\text { Consider nebulized (liposomal) } \\
\text { amikacin (IP and CP) }\end{array}$ \\
\hline M. avium complex & 3 drugs p.o.: macrolide $^{1}$, rifamycin ${ }^{2}$, ethambutol & $\begin{array}{l}\text { Amikacin (IP), consider nebulized } \\
\text { application; streptomycin (IP); } \\
\text { moxifloxacin; clofazimine }\end{array}$ \\
\hline M. kansasii & 3 drugs p.o.: rifampicin, ethambutol, isoniazid or a macrolide ${ }^{1}$ & Moxifloxacin \\
\hline M. malmoense & 3 drugs p.o.: macrolide $^{1}$, rifamycin ${ }^{2}$, ethambutol & Moxifloxacin \\
\hline M. simiae & $\begin{array}{l}\text { Amikacin (IP only): } \\
\text { plus } \\
2-3 \text { drugs p.o. (IP and CP) of the following: clofazimine, } \\
\text { macrolide }^{1} \text {, moxifloxacin, linezolid, trimethoprim/sulfamethoxazole }\end{array}$ & $\begin{array}{l}\text { Effective drug regimen for M. simiae } \\
\text { is unclear; in vitro DST shows } \\
\text { generally extensive drug-resistance }\end{array}$ \\
\hline M. xenopi & 3 drugs p.o.: macrolide $^{1}$, rifamycin ${ }^{2}$, ethambutol & Moxifloxacin \\
\hline
\end{tabular}

The choice of drugs for a treatment regimen should be based on the results of DST, if possible. $\mathrm{IP}=$ Intensive phase; $\mathrm{CP}=\mathrm{continuation}$ phase. ${ }^{1}$ Macrolide: azithromycin or clarithromycin. ${ }^{2}$ Rifamycin: rifampicin or rifabutin; if rifabutin is used, prefer azithromycin over clarithromycin.

Treatment durations:

- Recommendations for the duration of treatment have a low level of evidence

- IP, usually in M. abscessus therapy, occasionally in severe MAC or M. simiae-PD: 1-3 months or until sputum/bronchoalveolar lavage culture conversion; $\mathrm{CP}$, with orally available drugs only: 12 months beyond the time of sputum/bronchoalveolar lavage culture conversion

- For MAC-PD: in case of mild nodular bronchiectatic disease, an intermittent treatment 3 times per week with rifampicin, ethambutol, and a macrolide has been suggested

- General recommendation: if eradication of the NTM causing PD is not achieved by 6 months of therapy despite an appropriate choice of drugs, consider the option of surgery; if surgery is not possible, the goal of treatment, including the decision on the duration of treatment should focus on symptom control; short-course pulse treatments including i.v. therapy or continuous oral therapy/ inhalation therapy to suppress mycobacterial growth may be appropriate treatment options

A treatment duration of 12 months after sputum culture conversion is generally recommended for most NTM-PD [4], but it is very well possible that any standardized treatment recommendation is inadequate in the majority of cases [149]. Evidence-based individualization of treatment duration is not available, mainly due to the lack of biomarkers which would indicate treatment success and could guide physicians to discontinue antibiotics at a more appropriate time. It was shown in multidrug- and extensively drug-resistant TB that shortened therapy durations of 9-12 months can, under certain circumstances, be as effective as the recommended 20 -month treatment duration [150], and this could apply to diseases caused by other mycobacteria as well. Due to the complexity of the therapy of patients with NTM infections, treatment should be coordinated by physicians with sufficient experience and in constant consultation with respective reference centres. 
Table 4. Suggested research priorities to improve the management of NTM-PD

Priority measures

1. Development of interventions to prevent the development of NTM-PD in risk groups

2. Establishment of the relationship between in vitro DST and the in vivo efficacy of drugs used for the treatment of NTM-PD

3. Development of novel drugs for the treatment of NTM-PD

4. Development of adjunctive immunotherapies to improve treatment outcomes in NTM-PD

5. Identification of biomarkers to guide the decision on when to initiate NTM-PD therapy

6. Evaluation of synergistic/antagonistic effects of drugs used for combination antimycobacterial therapy

7. Identification of biomarkers that allow the prediction of treatment failure or success early in the course of antimycobacterial therapy

8. Ascertaining the value of therapeutic drug monitoring to optimize treatment outcomes in NTM-PD

9. Identification of biomarkers that allow individualization of the duration of treatment for NTM-PD

10. Finding an international consensus definition of treatment outcomes in NTM-PD

\section{Conclusions and Outlook}

NTM-PD belongs to the group of orphan diseases. Still, the demographic changes in Western societies might probably lead to a further increase in the incidence of NTM-PD in Europe and other industrialized regions. At present, clinical decisions affecting patients with NTM-PD heavily rely on expert opinion rather than on good clinical evidence. Care for affected patients can only be improved if research priorities (table 4) are recognized and addressed and better evidence for the management of NTM-PD is generated by basic and clinical research.
Evidence for the clinical management of patients with NTM-PD will require the collaboration of many colleagues from different clinical centres to generate reliable data together. Existing European networks such as the NTM-NET, a branch of the Tuberculosis Network European Trials group (TBNET), the ESCMYC [European Society of Clinical Microbiology and Infectious Diseases (ESCMID) Study Group on Mycobacteria], and the European Respiratory Society (ERS) Assembly for Respiratory Infections provide platforms for conducting such international multicentre studies, hopefully leading to a better understanding and management to improve the care for patients with NTM-PDs in the future.

\section{References}

1 Falkinham JO 3rd: Surrounded by mycobacteria: nontuberculous mycobacteria in the human environment. J Appl Microbiol 2009; 107:356-367.

2 LPSN: List of prokaryotic names with standing in nomenclature: genus Mycobacterium. 2014. www.bacterio.net.

-3 Falkinham JO 3rd: Environmental sources of nontuberculous mycobacteria. Clin Chest Med 2015;36:35-41.

4 Griffith DE, Aksamit T, Brown-Elliott BA, et al: An official ATS/IDSA statement: diagnosis, treatment, and prevention of nontuberculous mycobacterial diseases. Am J Respir Crit Care Med 2007;175:367-416.

5 Ricketts WM, O'Shaughnessy TC, van Ingen $\mathrm{J}$ : Human-to-human transmission of Mycobacterium kansasii or victims of a shared source? Eur Respir J 2014;44:1085-1087.
6 Aitken ML, Limaye A, Pottinger P, et al: Respiratory outbreak of Mycobacterium abscessus subspecies massiliense in a lung transplant and cystic fibrosis center. Am J Respir Crit Care Med 2012;185:231-232.

7 Bryant JM, Grogono DM, Greaves D, et al: Whole-genome sequencing to identify transmission of Mycobacterium abscessus between patients with cystic fibrosis: a retrospective cohort study. Lancet 2013;381:1551-1560.

8 van Ingen J, Hoefsloot W, Dekhuijzen PN, et al: The changing pattern of clinical Mycobacterium avium isolation in the Netherlands. Int J Tuberc Lung Dis 2010;14:1176-1180.

-9 Henry MT, Inamdar L, O'Riordain D, et al: Nontuberculous mycobacteria in non-HIV patients: epidemiology, treatment and response. Eur Respir J 2004;23:741-746.
10 McCallum AD, Watkin SW, Faccenda JF: Non-tuberculous mycobacterial infections in the Scottish Borders: identification, management and treatment outcomes - a retrospective review. J R Coll Physicians Edinb 2011;41: 294-303.

11 Ringshausen FC, Apel RM, Bange FC, et al: Burden and trends of hospitalisations associated with pulmonary non-tuberculous mycobacterial infections in Germany, 2005-2011. BMC Infect Dis 2013;13:231.

12 Marras TK, Chedore P, Ying AM, et al: Isolation prevalence of pulmonary non-tuberculous mycobacteria in Ontario, 1997-2003. Thorax 2007;62:661-666.

13 Park YS, Lee CH, Lee SM, et al: Rapid increase of non-tuberculous mycobacterial lung diseases at a tertiary referral hospital in South Korea. Int J Tuberc Lung Dis 2010;14:1069_ 1071. 
14 Brode SK, Daley CL, Marras TK: The epidemiologic relationship between tuberculosis and non-tuberculous mycobacterial disease: a systematic review. Int J Tuberc Lung Dis 2014;18:1370-1377.

-15 Marras TK, Mendelson D, Marchand-Austin A, et al: Pulmonary nontuberculous mycobacterial disease, Ontario, Canada, 19982010. Emerg Infect Dis 2013;19:1889-1891.

-16 Floto RA, Olivier KN, Saiman L, et al: US Cystic Fibrosis Foundation and European Cystic Fibrosis Society consensus recommendations for the management of non-tuberculous mycobacteria in individuals with cystic fibrosis: executive summary. Thorax 2016;71:88-90.

17 Floto RA, Olivier KN, Saiman L, et al: US Cystic Fibrosis Foundation and European Cystic Fibrosis Society consensus recommendations for the management of non-tuberculous mycobacteria in individuals with cystic fibrosis. Thorax 2016;71(suppl 1):i1-i22.

18 Lehmann KB, Neumann R: Atlas und Grundriss der Bakteriologie und Lehrbuch der speziellen bakteriologischen Diagnostik, ed 1. München, J.F. Lehmann, 1896.

19 Cayrou C, Turenne C, Behr MA, et al: Genotyping of Mycobacterium avium complex organisms using multispacer sequence typing. Microbiology 2010;156:687-694.

20 van Ingen J, Lindeboom JA, Hartwig NG, et al: Mycobacterium mantenii sp. nov., a pathogenic, slowly growing, scotochromogenic species. Int J Syst Evol Microbiol 2009;59: 2782-2787.

21 van Ingen J, Boeree MJ, Kosters K, et al: Proposal to elevate Mycobacterium avium complex ITS sequevar MAC-Q to Mycobacterium vulneris sp. nov. Int J Syst Evol Microbiol 2009;59:2277-2282.

22 Kim BJ, Math RK, Jeon CO, et al: Mycobacterium yongonense sp. nov., a slow-growing non-chromogenic species closely related to Mycobacterium intracellulare. Int J Syst Evol Microbiol 2013;63:192-199.

-23 van der Werf MJ, Ködmön C, KatalinićJanković $\mathrm{V}$, et al: Inventory study of nontuberculous mycobacteria in the European Union. BMC Infect Dis 2014;14:62.

24 Boyle DP, Zembower TR, Reddy S, et al: Comparison of clinical features, virulence, and relapse among Mycobacterium avium complex species. Am J Respir Crit Care Med 2015;191: 1310-1317.

$\checkmark 25$ Adékambi T, Berger P, Raoult D, et al: $r p o B$ gene sequence-based characterization of emerging non-tuberculous mycobacteria with descriptions of Mycobacterium bolletii sp. nov., Mycobacterium phocaicum sp. nov. and Mycobacterium aubagnense sp. nov. Int J Syst Evol Microbiol 2006;56:133-143.

26 Leao SC, Tortoli E, Euzéby JP, et al: Proposal that Mycobacterium massiliense and Mycobacterium bolletii be united and reclassified as Mycobacterium abscessus subsp. bolletii comb. nov., designation of Mycobacterium abscessus subsp. abscessus subsp. nov. and emended description of Mycobacterium ab- scessus. Int J Syst Evol Microbiol 2011;61: 2311-2313.

27 Koh WJ, Jeon K, Lee NY, et al: Clinical significance of differentiation of Mycobacterium massiliense from Mycobacterium abscessus. Am J Respir Crit Care Med 2011;183:405410 .

28 Benwill JL, Wallace RJ Jr: Mycobacterium abscessus: challenges in diagnosis and treatment. Curr Opin Infect Dis 2014;27:506-510.

29 van Ingen J, Griffith DE, Aksamit TA, et al: Pulmonary diseases caused by non-tuberculous mycobacteria. Eur Respir Monogr 2012; 58:25-37.

30 Hoefsloot W, van Ingen J, Andrejak C, et al: The geographic diversity of nontuberculous mycobacteria isolated from pulmonary samples: an NTM-NET collaborative study. Eur Respir J 2013;42:1604-1613.

31 Johnson MM, Odell JA: Nontuberculous mycobacterial pulmonary infections. J Thorac Dis 2014;6:210-220.

32 Prevots DR, Marras TK: Epidemiology of human pulmonary infection with nontuberculous mycobacteria: a review. Clin Chest Med 2015;36:13-34.

33 Lahiri A, Kneisel J, Kloster I, et al: Abundance of Mycobacterium avium ssp. hominissuis in soil and dust in Germany - implications for the infection route. Lett Appl Microbiol 2014; 59:65-70.

34 Martín-Casabona N, Bahrmand AR, Bennedsen J, et al: Non-tuberculous mycobacteria: patterns of isolation. A multi-country retrospective survey. Int J Tuberc Lung Dis 2004;8: 1186-1193.

35 Davies BS, Roberts CH, Kaul S, et al: Nontuberculous slow-growing mycobacterial pulmonary infections in non-HIV-infected patients in south London. Scand J Infect Dis 2012;44:815-819.

36 Prevots DR, Shaw PA, Strickland D, et al: Nontuberculous mycobacterial lung disease prevalence at four integrated health care delivery systems. Am J Respir Crit Care Med 2010;182:970-976.

37 Cassidy PM, Hedberg K, Saulson A, et al: Nontuberculous mycobacterial disease prevalence and risk factors: a changing epidemiology. Clin Infect Dis 2009;49:e124-e129.

38 Koh WJ, Kwon OJ, Jeon K, et al: Clinical significance of nontuberculous mycobacteria isolated from respiratory specimens in Korea. Chest 2006; 129:341-348.

39 Andrejak C, Thomsen VØ, Johansen IS, et al: Nontuberculous pulmonary mycobacteriosis in Denmark: incidence and prognostic factors. Am J Respir Crit Care Med 2010;181: 514-521.

40 Jankovic M, Samarzija M, Sabol I, et al: Geographical distribution and clinical relevance of non-tuberculous mycobacteria in Croatia. Int J Tuberc Lung Dis 2013;17:836-841.

41 Russell CD, Claxton P, Doig C, et al: Nontuberculous mycobacteria: a retrospective review of Scottish isolates from 2000 to 2010 . Thorax 2014;69:593-595.
42 Thomsen VØ, Andersen AB, Miörner H: Incidence and clinical significance of non-tuberculous mycobacteria isolated from clinical specimens during a 2-y nationwide survey. Scand J Infect Dis 2002;34:648-653.

43 Hoefsloot W, Boeree MJ, van Ingen J, et al: The rising incidence and clinical relevance of Mycobacterium malmoense: a review of the literature. Int J Tuberc Lung Dis 2008; 12:987993.

44 McShane PJ, Glassroth J: Pulmonary disease due to nontuberculous mycobacteria: current state and new insights. Chest 2015;148:15171527.

45 Chou MP, Clements ACA, Thomson RM: A spatial epidemiological analysis of nontuberculous mycobacterial infections in Queensland, Australia. BMC Infect Dis 2014; $14: 279$.

46 Moore JE, Kruijshaar ME, Ormerod LP, et al: Increasing reports of non-tuberculous mycobacteria in England, Wales and Northern Ireland, 1995-2006. BMC Public Health 2010; $10: 612$

47 van Ingen J, Bendien SA, de Lange WC, et al: Clinical relevance of non-tuberculous mycobacteria isolated in the Nijmegen-Arnhem region, The Netherlands. Thorax 2009;64:502506.

48 Panagiotou M, Papaioannou AI, Kostikas K, et al: The epidemiology of pulmonary nontuberculous mycobacteria: data from a general hospital in Athens, Greece, 2007-2013. Pulm Med 2014;2014:894976.

-49 Andrejak C, Nielsen R, Thomsen VØ, et al: Chronic respiratory disease, inhaled corticosteroids and risk of non-tuberculous mycobacteriosis. Thorax 2013;68:256-262.

50 Honda JR, Knight V, Chan ED: Pathogenesis and risk factors for nontuberculous mycobacterial lung disease. Clin Chest Med 2015;36 $1-11$.

51 Gommans EP, Even P, Linssen CF, et al: Risk factors for mortality in patients with pulmonary infections with non-tuberculous mycobacteria: a retrospective cohort study. Respir Med 2015;109:137-145.

52 Huang JH, Kao PN: Mycobacterium aviumintracellulare pulmonary infection in HIVnegative patients without preexisting lung disease diagnostic and management limitations. Chest 1999;115:1033-1040.

53 Lee BY, Kim S, Hong Y, et al: Risk factors for recurrence after successful treatment of $M y$ cobacterium avium complex lung disease. Antimicrob Agents Chemother 2015;59:29722977.

54 Kang HK, Park HY, Kim D, et al: Treatment outcomes of adjuvant resectional surgery for nontuberculous mycobacterial lung disease. BMC Infect Dis 2015;15:76

55 Carrillo MC, Patsios D, Wagnetz U, et al: Comparison of the spectrum of radiologic and clinical manifestations of pulmonary disease caused by Mycobacterium avium complex and Mycobacterium xenopi. Can Assoc Radiol J 2014;65:207-213. 
56 Ide S, Nakamura S, Yamamoto Y, et al: Epidemiology and clinical features of pulmonary nontuberculous mycobacteriosis in Nagasaki, Japan. PLoS One 2015; 10:e128304.

57 Maugein J, Dailloux M, Carbonnelle B, et al: Sentinel-site surveillance of Mycobacterium avium complex pulmonary disease. Eur Respir J 2005;26:1092-1096.

58 Dailloux M, Abalain ML, Laurain C, et al: Respiratory infections associated with nontuberculous mycobacteria in non-HIV patients. Eur Respir J 2006;28:1211-1215.

59 Griffith DE, Philley JV, Brown-Elliott BA, et al: The significance of Mycobacterium abscessus subspecies abscessus isolation during $M y$ cobacterium avium complex lung disease therapy. Chest 2015;147:1369-1375.

-60 Ellis SM, Hansell DM: Imaging of non-tuberculous (atypical) mycobacterial pulmonary infection. Clin Radiol 2002;57:661-669.

61 Moon SM, Park HY, Jeon K, et al: Clinical significance of Mycobacterium kansasii isolates from respiratory specimens. PLoS One 2015; 10:e0139621.

62 Yano Y, Kitada S, Mori M, et al: Pulmonary disease caused by rapidly growing mycobacteria: a retrospective study of 44 cases in Japan. Respiration 2013;85:305-311.

-63 Hoefsloot W, van Ingen J, de Lange WC, et al: Clinical relevance of Mycobacterium malmoense isolation in The Netherlands. Eur Respir J 2009;34:926-931.

64 Hollings NP, Wells AU, Wilson R, et al: Comparative appearances of non-tuberculous mycobacteria species: a CT study. Eur Radiol 2002;12:2211-2217.

65 van Ingen J, Boeree MJ, de Lange WC, et al: Mycobacterium xenopi clinical relevance and determinants, the Netherlands. Emerg Infect Dis 2008; 14:385-389.

66 Embil J, Warren P, Yakrus M: Pulmonary illness associated with exposure to Mycobacterium avium complex in hot tub water hypersensitivity pneumonitis or infection? Chest 1997;111:813-816.

67 Marras TK, Wallace RJ Jr, Koth LL, et al: Hypersensitivity pneumonitis reaction to $\mathrm{Myco}$ bacterium avium in household water. Chest 2005; 127:664-671.

68 Hankwitz PE, Cervia JS, Thomas CF, et al: Nontuberculous mycobacterial hypersensitivity pneumonitis related to a home shower: treatment and secondary prevention. BMJ Case Rep 2011;2011:bcr0620114360.

-69 Tillie-Leblond I, Grenouillet F, Reboux G, et al: Hypersensitivity pneumonitis and metalworking fluids contaminated by mycobacteria. Eur Respir J 2011;37:640-647.

70 Wallace RJ Jr, Zhang Y, Wilson RW, et al: Presence of a single genotype of the newly described species Mycobacterium immunogenum in industrial metalworking fluids associated with hypersensitivity pneumonitis. Appl Environ Microbiol 2002;68:5580-5584.

71 Hanak V, Kalra S, Aksamit TR, et al: Hot tub lung: presenting features and clinical course of 21 patients. Respir Med 2006;100:610-615.
72 Marchetti N, Criner K, Criner GJ: Characterization of functional, radiologic and lung function recovery post-treatment of hot tub lung. A case report and review of the literature. Lung 2004;182:271-277.

73 Rickman OB, Ryu JH, Fidler ME, et al: Hypersensitivity pneumonitis associated with $M y$ cobacterium avium complex and hot tub use. Mayo Clin Proc 2002;77:1233-1237.

74 Cappelluti E, Fraire AE, Schaefer OP: A case of 'hot tub lung' due to Mycobacterium avium complex in an immunocompetent host. Arch Intern Med 2003;163:845-848.

75 Wolinsky E: When is an infection disease? Rev Infect Dis 1981;3:1025-1027.

76 Management of opportunist mycobacterial infections: Joint Tuberculosis Committee Guidelines 1999. Subcommittee of the Joint Tuberculosis Committee of the British Thoracic Society. Thorax 2000;55:210-218.

77 van Ingen J: Microbiological diagnosis of nontuberculous mycobacterial pulmonary disease. Clin Chest Med 2015;36:43-54.

78 Wright PW, Wallace RJ Jr, Wright NW, et al: Sensitivity of fluorochrome microscopy for detection of Mycobacterium tuberculosis versus nontuberculous mycobacteria. J Clin Microbiol 1998;36:1046-1049.

79 Greco S, Girardi E, Navarra A, et al: Current evidence on diagnostic accuracy of commercially based nucleic acid amplification tests for the diagnosis of pulmonary tuberculosis. Thorax 2006;61:783-790.

80 Greco S, Rulli M, Girardi E, et al: Diagnostic accuracy of in-house PCR for pulmonary tuberculosis in smear-positive patients: metaanalysis and metaregression. J Clin Microbiol 2009;47:569-576.

81 Bicmen C, Gunduz AT, Coskun M, et al: Molecular detection and identification of $\mathrm{Myco-}$ bacterium tuberculosis complex and four clinically important nontuberculous mycobacterial species in smear-negative clinical samples by the genotype mycobacteria direct test. J Clin Microbiol 2011;49:2874-2878.

82 Wang HY, Bang H, Kim S, et al: Identification of Mycobacterium species in direct respiratory specimens using reverse blot hybridisation assay. Int J Tuberc Lung Dis 2014;18:11141120.

83 Medical Microbiology - Diagnosis of Tuberculosis - Part 3: Detection of Mycobacteria by Culture Methods; text in German and English. Berlin, Beuth, 2011.

84 Richter E, Beer J, Diel R, Hillemann D, Hoffmann H, Klotz M, Mauch H, Rüsch-Gerdes S: MiQ 5: Tuberkulose Mykobakteriose, ed 2. Berlin, Elsevier, 2010.

85 Sorlozano A, Soria I, Roman J, et al: Comparative evaluation of three culture methods for the isolation of mycobacteria from clinical samples. J Microbiol Biotechnol 2009;19: 1259-1264.

86 Piersimoni C, Nista D, Bornigia S, et al: Unreliable detection of Mycobacterium xenopi by the nonradiometric Bactec MGIT 960 culture system. J Clin Microbiol 2009;47:804-806.
87 Lindeboom JA, Bruijnesteijn van Coppenraet LE, van Soolingen D, et al: Clinical manifestations, diagnosis, and treatment of Mycobacterium haemophilum infections. Clin Microbiol Rev 2011;24:701-717.

88 Santos M, Gil-Brusola A, Escandell A, et al: Mycobacterium genavense infections in a tertiary hospital and reviewed cases in nonHIV patients. Patholog Res Int 2014;2014: 371370.

89 Brent AJ, Mugo D, Musyimi R, et al: Performance of the MGIT TBc identification test and meta-analysis of MPT64 assays for identification of the Mycobacterium tuberculosis complex in liquid culture. J Clin Microbiol 2011;49:4343-4346.

90 Buchan BW, Riebe KM, Timke M, et al: Comparison of MALDI-TOF MS with HPLC and nucleic acid sequencing for the identification of Mycobacterium species in cultures using solid medium and broth. Am J Clin Pathol 2014;141:25-34.

91 Adams LL, Salee P, Dionne K, et al: A novel protein extraction method for identification of mycobacteria using MALDI-ToF MS. J Microbiol Methods 2015;119:1-3.

92 Kasai H, Ezaki T, Harayama S: Differentiation of phylogenetically related slowly growing mycobacteria by their $\operatorname{gyr} B$ sequences. J Clin Microbiol 2000;38:301-308.

93 Bastian S, Veziris N, Roux AL, et al: Assessment of clarithromycin susceptibility in strains belonging to the Mycobacterium abscessus group by erm(41) and $\mathrm{rrl}$ sequencing. Antimicrob Agents Chemother 2011;55:775781.

94 van Ingen J, Boeree MJ, van Soolingen D, et al: Resistance mechanisms and drug susceptibility testing of nontuberculous mycobacteria. Drug Resist Updat 2012;15:149-161.

95 M24-A2: Susceptibility Testing of Mycobacteria, Nocardiae, and Other Aerobic Actinomycetes; Approved Standard, ed 2, vol 31, No 5. Wayne, National Committee for Clinical Laboratory Standards, 2011.

96 European Committee on Antimicrobial Susceptibility Testing: Breakpoint tables for interpretation of MICs and zone diameters, version 5.0. 2015. http://www.eucast.org.

97 Schönfeld N, Haas W, Richter E, et al: Recommendations for diagnosis and treatment of nontuberculous mycobacterioses of the German Central Committee against Tuberculosis and the German Respiratory Society (in German). Pneumologie 2013;67:605633.

98 Plotinsky RN, Talbot EA, von Reyn CF: Proposed definitions for epidemiologic and clinical studies of Mycobacterium avium complex pulmonary disease. PLoS One 2013;8: e77385.

99 Egelund EF, Fennelly KP, Peloquin CA: Medications and monitoring in nontuberculous mycobacteria infections. Clin Chest Med 2015;36:55-66 
100 Klemens SP, Cynamon MH: Activities of 113 Koh WJ, Jeong BH, Jeon K, et al: Therapeuazithromycin and clarithromycin against nontuberculous mycobacteria in beige mice. Antimicrob Agents Chemother 1994; 38:1455-1459.

101 Andrejak C, Almeida DV, Tyagi S, et al: Improving existing tools for Mycobacterium xenopi treatment: assessment of drug combinations and characterization of mouse models of infection and chemotherapy. J Antimicrob Chemother 2013;68:659-665.

102 Miwa S, Shirai M, Toyoshima M, et al: Efficacy of clarithromycin and ethambutol for Mycobacterium avium complex pulmonary disease. A preliminary study. Ann Am Thorac Soc 2014;11:23-29.

103 Kobashi Y, Matsushima T, Oka M: A double-blind randomized study of aminoglycoside infusion with combined therapy for pulmonary Mycobacterium avium complex disease. Respir Med 2007;101:130-138.

104 van Ingen J, Verhagen AF, Dekhuijzen PN, et al: Surgical treatment of non-tuberculous mycobacterial lung disease: strike in time. Int J Tuberc Lung Dis 2010;14:99-105.

105 Wassilew N, Lange C: Cure not possible, by definition. Am J Respir Crit Care Med 2015; 192:1255-1256.

106 Horsburgh CR Jr, Mason UG 3rd, Heifets $\mathrm{LB}$, et al: Response to therapy of pulmonary Mycobacterium avium-intracellulare infection correlates with results of in vitro susceptibility testing. Am Rev Respir Dis 1987; 135:418-421.

107 Heifets L, Mor N, Vanderkolk J: Mycobacterium avium strains resistant to clarithromycin and azithromycin. Antimicrob Agents Chemother 1993;37:2364-2370.

108 Wallace RJ Jr, Brown BA, Griffith DE, et al: Clarithromycin regimens for pulmonary Mycobacterium avium complex. The first 50 patients. Am J Respir Crit Care Med 1996; 153:1766-1772.

109 Brown-Elliott BA, Iakhiaeva E, Griffith DE, et al: In vitro activity of amikacin against isolates of Mycobacterium avium complex with proposed MIC breakpoints and finding of a 16S rRNA gene mutation in treated isolates. J Clin Microbiol 2013;51:33893394.

110 Dunne M, Fessel J, Kumar P, et al: A randomized, double-blind trial comparing azithromycin and clarithromycin in the treatment of disseminated Mycobacterium avium infection in patients with human immunodeficiency virus. Clin Infect Dis 2000; 31:1245-1252.

-111 Magis-Escurra C, Alffenaar JW, Hoefnagels I, et al: Pharmacokinetic studies in patients with nontuberculous mycobacterial lung infections. Int J Antimicrob Agents 2013;42: 256-261.

112 van Ingen J, Egelund EF, Levin A, et al: The pharmacokinetics and pharmacodynamics of pulmonary Mycobacterium avium complex disease treatment. Am J Respir Crit Care Med 2012;186:559-565. tic drug monitoring in the treatment of $M y$ cobacterium avium complex lung disease. Am J Respir Crit Care Med 2012;186:797802.

114 Schluger NW: Treatment of pulmonary $M y$ cobacterium avium complex infections: do drug levels matter? Am J Respir Crit Care Med 2012;186:710-711.

115 Shafran SD, Singer J, Zarowny DP, et al: Determinants of rifabutin-associated uveitis in patients treated with rifabutin, clarithromycin, and ethambutol for Mycobacterium avium complex bacteremia: a multivariate analysis. Canadian HIV Trials Network Protocol 010 Study Group. J Infect Dis 1998; 177:252-255.

116 Apseloff G, Foulds G, LaBoy-Goral L, et al: Comparison of azithromycin and clarithromycin in their interactions with rifabutin in healthy volunteers. J Clin Pharmacol 1998; 38:830-835.

117 Jeong BH, Jeon K, Park HY, et al: Intermittent antibiotic therapy for nodular bronchiectatic Mycobacterium avium complex lung disease. Am J Respir Crit Care Med 2015; 191:96-103.

118 Wallace RJ Jr, Brown-Elliott BA, McNulty S, et al: Macrolide/azalide therapy for nodular/ bronchiectatic mycobacterium avium complex lung disease. Chest 2014;146:276-282.

119 van Ingen J, Ferro BE, Hoefsloot W, et al: Drug treatment of pulmonary nontuberculous mycobacterial disease in HIV-negative patients: the evidence. Expert Rev Anti Infect Ther 2013;11:1065-1077.

120 Field SK, Cowie RL: Treatment of Mycobacterium avium-intracellulare complex lung disease with a macrolide, ethambutol, and clofazimine. Chest 2003;124:1482-1486.

121 Roussel G, Igual J: Clarithromycin with minocycline and clofazimine for Mycobacterium avium intracellulare complex lung disease in patients without the acquired immune deficiency syndrome. GETIM. Groupe d'Etude et de Traitement des Infections a Mycobacteries. Int J Tuberc Lung Dis 1998;2:462-470.

122 Ferro BE, van Ingen J, Wattenberg M, et al: Time-kill kinetics of slowly growing mycobacteria common in pulmonary disease. J Antimicrob Chemother 2015;70:28382843.

123 Olivier KN, Shaw PA, Glaser TS, et al: Inhaled amikacin for treatment of refractory pulmonary nontuberculous mycobacterial disease. Ann Am Thorac Soc 2014;11:3035.

124 Rose SJ, Neville ME, Gupta R, et al: Delivery of aerosolized liposomal amikacin as a novel approach for the treatment of nontuberculous mycobacteria in an experimental model of pulmonary infection. PLoS One 2014;9:e108703.

125 van Ingen J, Totten SE, Helstrom NK, et al: In vitro synergy between clofazimine and amikacin in treatment of nontuberculous mycobacterial disease. Antimicrob Agents Chemother 2012;56:6324-6327.

126 Brown-Elliott BA, Crist CJ, Mann LB, et al: In vitro activity of linezolid against slowly growing nontuberculous mycobacteria. Antimicrob Agents Chemother 2003;47:17361738.

127 Jo KW, Kim S, Lee JY, et al: Treatment outcomes of refractory MAC pulmonary disease treated with drugs with unclear efficacy. J Infect Chemother 2014;20:602-606.

128 Winthrop KL, Ku JH, Marras TK, et al: The tolerability of linezolid in the treatment of nontuberculous mycobacterial disease. Eur Respir J 2015;45:1177-1179.

129 Pezzia W, Raleigh JW, Bailey MC, et al: Treatment of pulmonary disease due to Mycobacterium kansasii: recent experience with rifampin. Rev Infect Dis 1981;3:10351039.

130 Ahn CH, Lowell JR, Ahn SS, et al: Shortcourse chemotherapy for pulmonary disease caused by Mycobacterium kansasii. Am Rev Respir Dis 1983;128:1048-1050.

131 Mycobacterium kansasii pulmonary infection: a prospective study of the results of nine months of treatment with rifampicin and ethambutol. Research Committee, British Thoracic Society. Thorax 1994;49:442-445.

132 Jenkins PA, Campbell IA, Banks J, et al: Clarithromycin vs ciprofloxacin as adjuncts to rifampicin and ethambutol in treating opportunist mycobacterial lung diseases and an assessment of Mycobacterium vaccae immunotherapy. Thorax 2008;63:627-634.

133 Research Committee of the British Thoracic Society: First randomised trial of treatments for pulmonary disease caused by $M$. avium intracellulare, M. malmoense, and M. xenopi in HIV negative patients: rifampicin, ethambutol and isoniazid versus rifampicin and ethambutol. Thorax 2001;56:167-172.

134 Valero G, Moreno F, Graybill JR: Activities of clarithromycin, ofloxacin, and clarithromycin plus ethambutol against Mycobacterium simiae in murine model of disseminated infection. Antimicrob Agents Chemother 1994;38:2676-2677.

135 van Ingen J, Totten SE, Helstrom NK, et al: In vitro synergy between clofazimine and amikacin in treatment of nontuberculous mycobacterial disease. Antimicrob Agents Chemother 2012;56:6324-6327.

136 Kasperbauer SH, De Groote MA: The treatment of rapidly growing mycobacterial infections. Clin Chest Med 2015;36:67-78.

137 Nessar R, Cambau E, Reyrat JM, et al: $M y$ cobacterium abscessus: a new antibiotic nightmare. J Antimicrob Chemother 2012; 67:810-818.

138 Ripoll F, Pasek S, Schenowitz C, et al: Non mycobacterial virulence genes in the genome of the emerging pathogen Mycobacterium abscessus. PLoS One 2009;4:e5660.

139 Ingen JV, Kuijper EJ: Drug susceptibility testing of nontuberculous mycobacteria. Future Microbiol 2014;9:1095-1110. 
140 Jarand J, Levin A, Zhang L, et al: Clinical and microbiologic outcomes in patients receiving treatment for Mycobacterium abscessus pulmonary disease. Clin Infect Dis 2011;52: 565-571.

141 Jeon K, Kwon OJ, Lee NY, et al: Antibiotic treatment of Mycobacterium abscessus lung disease: a retrospective analysis of 65 patients. Am J Respir Crit Care Med 2009;180: 896-902.

142 Koh W-J, Jeon K, Lee NY, et al: Clinical significance of differentiation of Mycobacterium massiliense from Mycobacterium abscessus. Am J Respir Crit Care Med 2011;183: 405-410.

143 Harada T, Akiyama Y, Kurashima A, et al: Clinical and microbiological differences between Mycobacterium abscessus and Myco- bacterium massiliense lung diseases. J Clin Microbiol 2012;50:3556-3561.

144 van Ingen J: Treatment of pulmonary disease caused by non-tuberculous mycobacteria. Lancet Respir Med 2015;3:179-180.

145 Shen GH, Wu BD, Hu ST, et al: High efficacy of clofazimine and its synergistic effect with amikacin against rapidly growing mycobacteria. Int J Antimicrob Agents 2010; 35:400-404.

146 Philley JV, Wallace RJ Jr, Benwill JL, et al: Preliminary results of bedaquiline as salvage therapy for patients with nontuberculous mycobacterial lung disease. Chest 2015;148: 499-506.

147 Choi GE, Shin SJ, Won CJ, et al: Macrolide treatment for Mycobacterium abscessus and Mycobacterium massiliense infection and inducible resistance. Am J Respir Crit Care Med 2012;186:917-925.

48 Maurer FP, Castelberg C, Quiblier C, et al: Erm(41)-dependent inducible resistance to azithromycin and clarithromycin in clinical isolates of Mycobacterium abscessus. J Antimicrob Chemother 2014;69:1559-1563.

149 Heyckendorf J, Olaru ID, Ruhwald M, et al: Getting personal perspectives on individualized treatment duration in multidrug-resistant and extensively drug-resistant tuberculosis. Am J Respir Crit Care Med 2014; 190:374-383.

50 Van Deun A, Maug AK, Salim MA, et al: Short, highly effective, and inexpensive standardized treatment of multidrug-resistant tuberculosis. Am J Respir Crit Care Med 2010;182:684-692. 